DISTRIBUTION STATEMENT A. Approved for public release; distribution is unlimited.

\title{
Modeling Statistics of Fish Patchiness and Predicting Associated Influence on Statistics of Acoustic Echoes
}

\author{
Timothy K. Stanton \\ Applied Ocean Physics and Engineering Department \\ Woods Hole Oceanographic Institution \\ Bigelow 201, MS \#11 \\ Woods Hole, MA 02543 \\ phone: (508) 289-2757 fax:(508) 457-2194ｅmail: tstanton@whoi.edu \\ Daniel Grunbaum \\ School of Oceanography \\ University of Washington \\ Seattle, WA 98195-7940 \\ email: grunbaum@ocean.washintgon.edu \\ Thomas C. Weber \\ Center for Coastal and Ocean Mapping \\ Jere A. Chase Ocean Engineering Laboratory \\ University of New Hampshire \\ Durham, NH 03824 \\ email:weber@ccom.unh.edu \\ Award Number: N00014-11-1-0147 \\ http://www.whoi.edu/people/tstanton
}

\section{LONG-TERM GOALS}

To accurately describe the statistics of acoustic echoes due to the presence of fish, especially in the case of a long-range active sonar. Toward this goal, fundamental advances in the understanding of fish behavior, especially in aggregations, will be made under conditions relevant to the echo statistics problem.

\section{OBJECTIVES}

To develop new models of behavior of fish aggregations, including the fission/fusion process, and to describe the echo statistics associated with the random fish behavior using existing formulations of echo statistics.

\section{APPROACH}

The research begins with development of new advanced models of fish behavior inspired by, and grounded by, existing 3-D images of fish aggregations. These images are derived by multi-beam acoustic systems. Key parameters to be observed and modeled are the fission/fusion rate of the aggregations. Concurrent with the modeling of fish behavior, statistics of fish aggregations, as they 


\section{Report Documentation Page}

Form Approved

OMB No. 0704-0188

Public reporting burden for the collection of information is estimated to average 1 hour per response, including the time for reviewing instructions, searching existing data sources, gathering and maintaining the data needed, and completing and reviewing the collection of information. Send comments regarding this burden estimate or any other aspect of this collection of information,

including suggestions for reducing this burden, to Washington Headquarters Services, Directorate for Information Operations and Reports, 1215 Jefferson Davis Highway, Suite 1204, Arlington

VA 22202-4302. Respondents should be aware that notwithstanding any other provision of law, no person shall be subject to a penalty for failing to comply with a collection of information if it

does not display a currently valid OMB control number.

1. REPORT DATE

30 SEP 2013

4. TITLE AND SUBTITLE

Modeling Statistics of Fish Patchiness and Predicting Associated Influence on Statistics of Acoustic Echoes

6. $\operatorname{AUTHOR}(\mathrm{S})$

7. PERFORMING ORGANIZATION NAME(S) AND ADDRESS(ES)

Woods Hole Oceanographic Institution,Applied Ocean Physics and Engineering Department,Woods Hole,MA,02543

9. SPONSORING/MONITORING AGENCY NAME(S) AND ADDRESS(ES)

12. DISTRIBUTION/AVAILABILITY STATEMENT

Approved for public release; distribution unlimited

13. SUPPLEMENTARY NOTES

14. ABSTRACT

15. SUBJECT TERMS

16. SECURITY CLASSIFICATION OF:

a. REPORT

unclassified b. ABSTRACT

unclassified c. THIS PAGE

unclassified
17. LIMITATION OF ABSTRACT

Same as

Report (SAR)
3. DATES COVERED

00-00-2013 to 00-00-2013

5a. CONTRACT NUMBER

5b. GRANT NUMBER

5c. PROGRAM ELEMENT NUMBER

5d. PROJECT NUMBER

5e. TASK NUMBER

5f. WORK UNIT NUMBER

8. PERFORMING ORGANIZATION REPORT NUMBER

10. SPONSOR/MONITOR'S ACRONYM(S)

11. SPONSOR/MONITOR'S REPORT NUMBER(S) 
become available, will be incorporated into an existing general formulation for echo statistics. The results of this echo statistics model will, in turn, help to drive further development of the fish behavior model. In parallel to these efforts, other groups in the BRC will be conducting measurements. The Stanton/Weber/Grunbaum group participated in planning those experiments which may have a component that reveals key aspects of fish behavior and, in turn, contribute to the modeling.

Stanton oversees the entire project as well as works with Weber on incorporating fish behavior models into echo statistics predictions. Weber also analyzes images of fish aggregations that he and others have collected. Grunbaum develops the fish behavior models. The work also involves informal collaborations with Chris Wilson of NOAA Alaska Fisheries and Ben Jones of NPS.

\section{WORK COMPLETED}

Major milestones were reached this year including one new paper that was submitted to a refereed journal and a previously submitted paper that was revised. The new paper involved predicting echo statistics due to a long-range sonar insonifying schools of fish in an ocean waveguide. The paper under revision involved comparing past models of fish behavior with 3-D multi-beam acoustic data. In addition, our recently developed fish behavior model (from last year) was parameterized with laboratory and ocean data; and parameters of fish behavior (fission/fusion rates) were extracted from recently collected 3-D multi-beam acoustic data.

\section{Comparing previous competing behavior models with 3-D multi-beam data}

Last year on this project, previous competing models were compared with published 3-D multi-beam data. The Niwa and Anderson models were compared with 3-D multi-beam data collected by Paramo and Gerlotto. The data were consistent with the Anderson model in that both the data and model had a mode in the statistics of fish school dimensions (whereas the Niwa model does not have a mode). Based on the reviews of the submitted paper, we have revised the analysis by also analyzing the statistics of the volume of the fish schools (which is uniquely provided by the multi-beam data) (Bhatia et al., under revision). The statistics of the fish school volume are also consistent with the Anderson model. This new observation strengthens last year's conclusions that the assumptions in the Anderson model on fish dynamics apply to these fish - the rate at which fish exit the school is proportional to school size.

\section{Parameterizing and optimizing recent model for fish school behavior}

Last year on this project, we developed and published the first model of social animal aggregation behaviors to incorporate explicit spatial memory and cognitive responses to neighbors. This year, we focused on estimating parameters of the new model using individual-level and population-level fish schooling data. The individual-level data are 10-minute-long 3-dimensional-trajectory sequences of all individuals within small schooling and milling aggregations in the laboratory. The population-level data were collected in field surveys of Alaska pollock being analyzed by Weber and Stanton. The model and its application to schooling analysis involve a relatively large number of parameters, which must be constrained and optimized with respect to the available data. Therefore, much of the effort this year also involved building computational and statistical machinery to execute this optimization. Both the number of parameters and the relatively high computational demands of large-scale spatiallyexplicit schooling models make effectiveness and efficiency key to successful parameter-fitting. This year's principal modeling accomplishments are summarized: 
(a) We reanalyzed individual-level fish trajectory data to comprehensively characterize individual positions and movements within groups and to quantify neighbor-neighbor turning and acceleration responses. We developed new computational machinery for parameter-fitting behavioral models to our individual-level fish trajectory data. We conducted large-scale numerical optimization to obtain best-fitting behavioral response zones for short-memory cases; we are currently extending our analysis to long-memory cases. We conducted simulations using the best-fit behaviors to assess the degree to which they reflect the observed behaviors from which they were extracted (Fig. 1).

(b) We developed model computer code to embed observed fish trajectories into the new cognitive schooling model. This enables us to conduct simulations in which modeled fish respond to actual observed fish behavior. This also enables us to assess model behaviors by replacing one or more members of a real fish aggregation with a simulated counterpart, and quantifying the degree to which position, movement and neighbor-neighbor responses are statistically consistent with the true behavior.

(c) We developed model computer code to identify and characterize schools in the output of the cognitive schooling model (Fig. 2). Identification of schools is based largely on mathematical morphology analysis techniques for "segmentation" of features in images. Densities and edge characteristics of groups are used to delineate them from surrounding non-group distributions. This analytical machinery is a key tool in constraining model parameters to field surveys of fish schools that quantify distributions of group size, shape and separation distance.

\section{Extracting fission/fusion parameters from high resolution images of fish aggregations derived from acoustic multibeam data}

This year we focused on analyzing and interpreting a subset of data collected in the summer of 2012 using a Simrad ME70 multibeam echosounder data as part of the NOAA Alaska Fisheries Science Center acoustic/trawl walley pollock survey in the Gulf of Alaska. The data concerned repeated transects over the same discrete aggregations of pollock that were approximately $1 \mathrm{nmi}$ long and collected at approximately 15 minute intervals. These data are being analyzed to extract metrics describing morphological changes in the fish aggregations that can be used to tune or ground-truth behavioral models. Metrics of particular interest include the size-dependent group speed and bounds on the rate at which aggregations appear to split (a 'fusion' event) or recombine (a 'fission' event).

An example showing six passes (approximately 1:05 hours) over an aggregation of fish is shown in Figure 3 (upper panel). During the first two passes (A and B), the aggregations have approximately the same volume and volumetric scattering strength. The aggregation then splits into two subgroups over the subsequent two passes $(\mathrm{C} 1 / \mathrm{C} 2$ and D1/D2). The split-aggregation then appears to recombine between the fourth and fifth passes, and remains mostly together on a sixth pass. During these passes, the group (or subgroup) volumes vary between $12,000 \mathrm{~m}^{3}$ (C2) and $55,000 \mathrm{~m}^{3}$ (F) and have speeds ranging from $0.9 \mathrm{~cm} / \mathrm{s}(\mathrm{B}$ to $\mathrm{C} 2)$ and $6.8 \mathrm{~cm} / \mathrm{s}$ (D1 to E). The multibeam scattering strength measurements suggest that there are $\mathrm{O}(1)$ fish per cubic meter.

The analysis of data such as those shown in Figure 3 (lower panel) provide quantitative metrics describing fish group behavior (e.g., the speed at which groups of various sizes move) that are rarely seen outside of laboratory settings. The detection of fusion/fision events in fish groups is rare even for 
repeat pass surveys, but when they are observed they help to bound the space/time scales at which information is passed (or remains coherent) within the school.

\section{Echo statistics due to various aggregations of fish detected by a mid-frequency long-range sonar}

Last year, as part of the goal of characterizing echo statistics due to aggregations of fish detected by a long-range sonar, we made calculations involving several simple cases as illustrated in last year's

report. Reverberation was predicted for a mid-frequency sonar deployed near the surface in an ocean waveguide (many $\mathrm{km}$ long). Two sets of calculations were made, one with the community standard PE code, and the other with a numerically efficient code that we developed. Several example calculations were made with 1, 2, 5, and 10 identical small aggregations of fish in the waveguide. The calculations demonstrate the degree to which the statistics are non-Rayleigh, with the "tail" of the echo probability density function (PDF) increasing with decreasing numbers of aggregations.

This year, those results, as well as predictions involving a wide range of realistic waveguide conditions (randomized environment with internal waves), were completed and submitted for publication (Jones et al., submitted).

\section{RESULTS}

Our key advances this year involved multiple aspects of grounding our new theoretical behavior model with experimental data. Recently collected 3-D data concerning a time series of aggregations of fish are providing a rare assessment of the fission and fusion rates of fish and provide critical information on fish behavior in their natural environment. These group-level ocean data, coupled with our laboratory data involving individual fish, are being instrumental in enabling the model (through experimental parameterization) to make realistic predictions.

Our results are further illustrating the great complexity of fish behavior, which is important at scales relevant to both the individual and group level. Our simulations show that our cognitive schooling model can exhibit a wide variety of population-level behaviors, and these are typically strongly sensitive to the timescale of spatial memory. For example, the modal group size shifts to larger groups when the memory timescale is increased, all other parameters held constant. The strong effects of spatial memory, cognitive algorithms and memory timescale on group-size distributions and other population-level characteristics suggest that mechanistic models are likely to have better predictive capabilities if they incorporate cognitive behavioral algorithms. Behavioral parameters that we are extracting from laboratory and field data are helping us to ground key aspects of the model. Our predictions of echo statistics due to a long-range sonar for simple aggregations of fish show that the degree to which the echoes are non-Rayleigh depends upon the number of fish (or fish aggregations). The behavior modeling is working toward making predictions of echo statistics due to realistic aggregations of fish.

\section{IMPACT/APPLICATIONS}

The modeling and observations of fish behavior represent an advancement of the fundamental understanding of fish behavior. Integrating the data with the model is creating a powerful tool for making realistic predictions of fish behavior. The modeling of echo statistics from a mid-frequency sonar with several simplistic examples of fish aggregations demonstrates the fish clutter characteristics 
relevant to Navy ASW applications. Once the advanced behavior model is incorporated into the echo statistics model, we will have a significant tool for predicting sonar performance associated with the presence of fish.

\section{TRANSITIONS}

The 3-D fish shoal data, provided by NOAA Fisheries and analyzed in this project, were the basis for a transition this year of the HiFAST biologic simulations in the CASE (NAVAIR) sonar trainer. In addition, transition of the shoal data into the SAST ACB15 (NAVSEA) sonar trainer was approved this year and scheduled to be executed in a future year.

\section{RELATED PROJECTS}

Parts of this project fed into the ONR HIFAST FNC program this year in which fish echoes were simulated for use in Navy sonar trainers (SAST-NAVSEA and CASE-NAVAIR) (see "Transitions" above). The 3-D multi-beam data involving fish shoals from the Eastern Bering Sea, provided by NOAA Fisheries and analyzed in this project, were used in the HiFAST program to predict echoes from shoals.

\section{PUBLICATIONS}

Jones, B.A., J.A. Colosi, and T.K. Stanton, "Echo statistics of individuals and aggregations of scatterers in the water column of a random, oceanic waveguide," submitted to J. Acoust. Soc. Am [submitted, refereed]

Bhatia, S., T.K. Stanton, J. Paramo, and F. Gerlotto (under revision), "Modeling statistics of fish school dimensions using 3-D data from a multibeam sonar," under revision based on last year's submission to J. Theor. Bio. [submitted, refereed] 

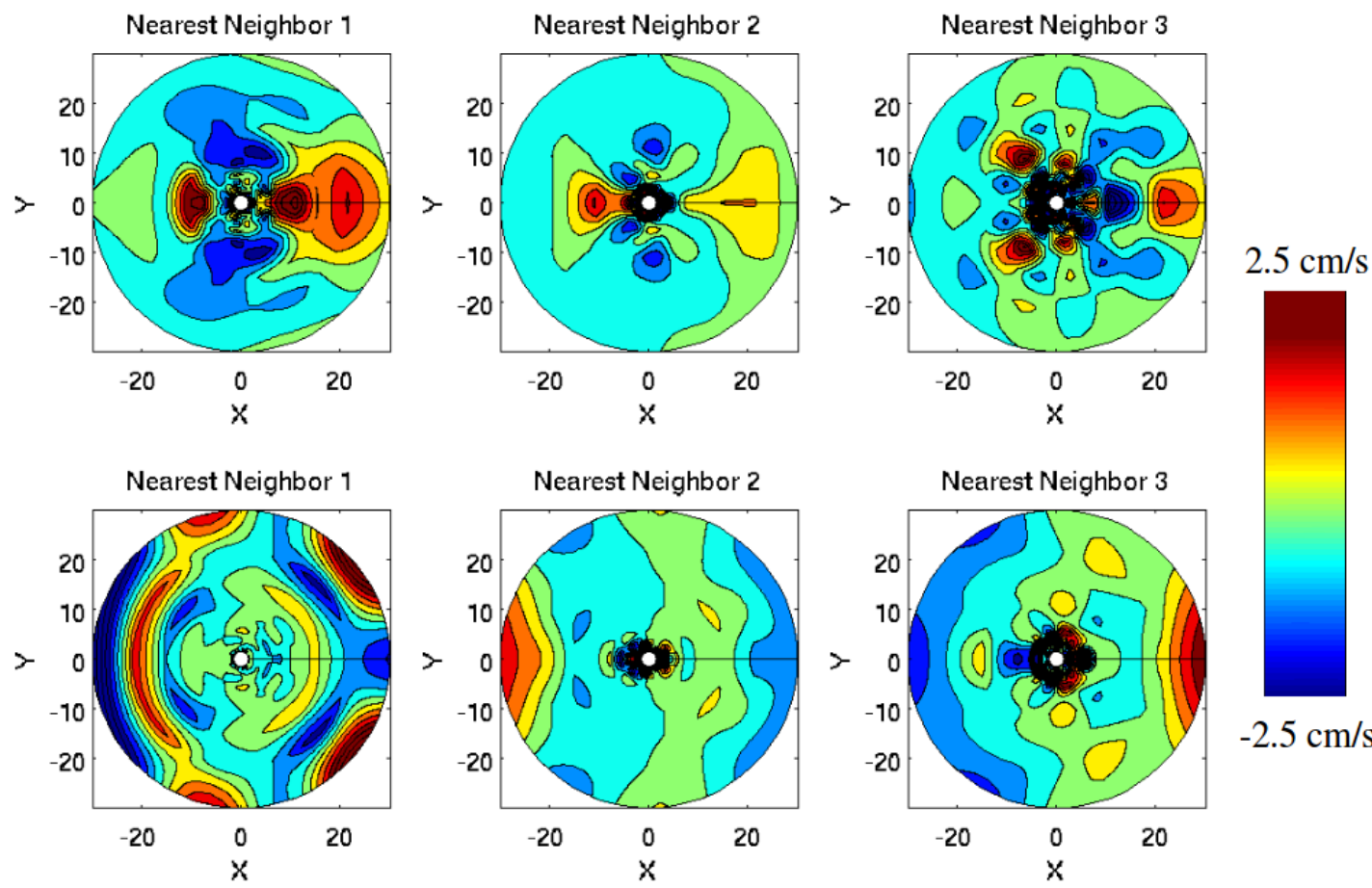

$-2.5 \mathrm{~cm} / \mathrm{s}$

Figure 1. Numerical optimization of behavioral responses to neighbors in a diffuse milling group (top row) and a polarized group (bottom row). The graphics are "heat maps" representing speed adjustments in response to neighbors that minimize errors in predicted movements relative to 10minute 3-dimensional trajectories of Giant Danios in the laboratory (Viscido et al. 2004, 2005, 2007). In the optimization, fish are assumed to respond to the nearest three neighbors within $30 \mathrm{~cm}$. Colors represent changes relative to the overall average observed swimming speed $(\mathrm{red}=+2.5 \mathrm{~cm}$ per sec.; blue = -2.5 cm per sec). The focal fish is at the center of each circle; movement is to the right. For example, the top left graphic shows that if a focal fish in a diffuse milling group has a nearest neighbor $20 \mathrm{~cm}$ ahead, its speed is predicted to increase by approximately $2.5 \mathrm{~cm} / \mathrm{s}$. The bottom left graphic shows that a focal fish in a polarized group has a nearest neighbor in the same position, its speed is predicted to decrease by approximately $1.5 \mathrm{~cm} / \mathrm{s}$. 


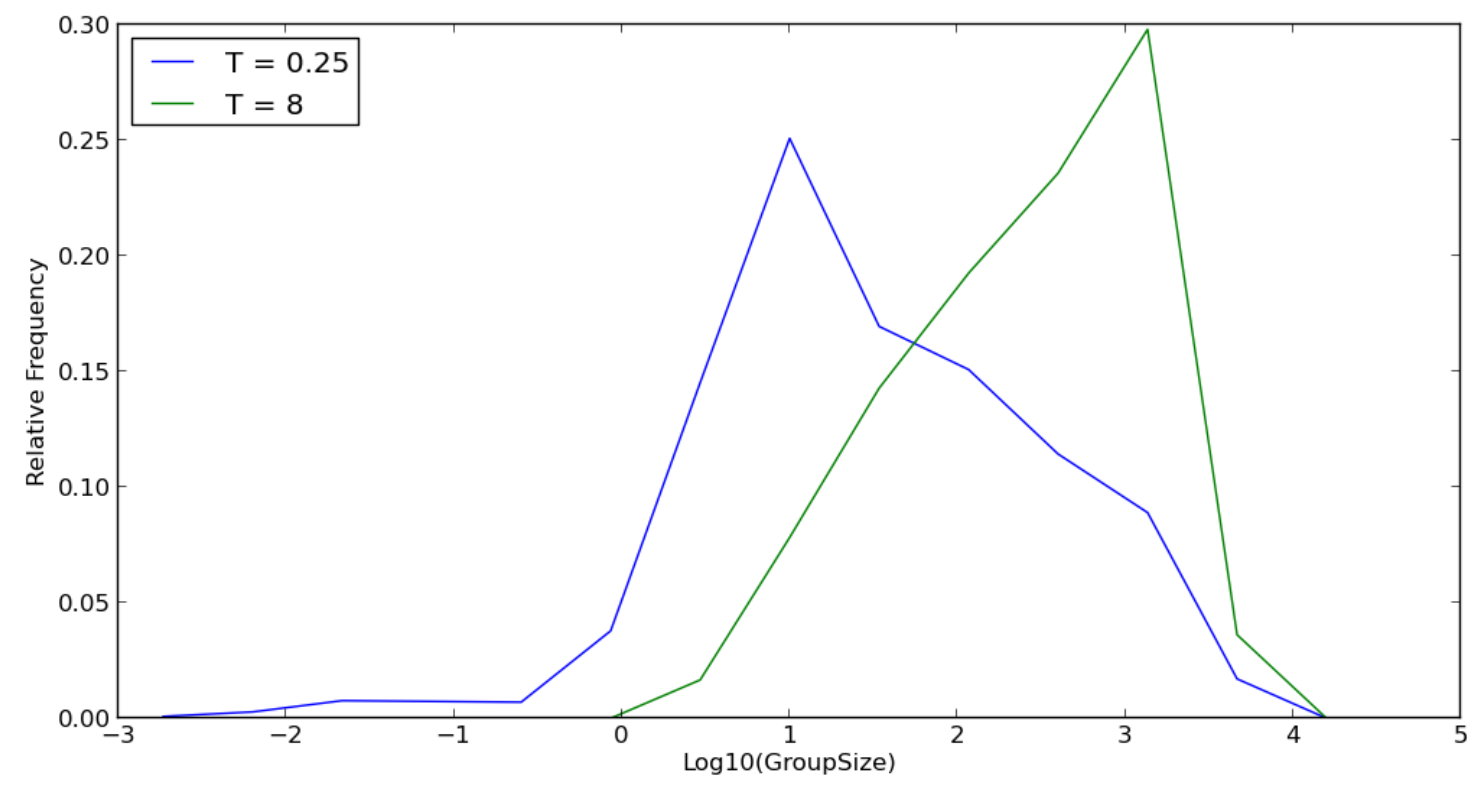

Figure 2. An example of group size distributions from school segmentation in the cognitive schooling model using mathematical morphology techniques. Size is quantified as the total number of fish within separate schools. Units are arbitrary (rescaled and nondimensionalized from physical units). This plot illustrates the strong effect of spatial memory on group-and population-level dynamics: Here, a shift from an effectively "short" memory $(T<1)$ to an effectively "long" memory $(T>1)$ results in an increase in modal group size of roughly two orders of magnitude. 

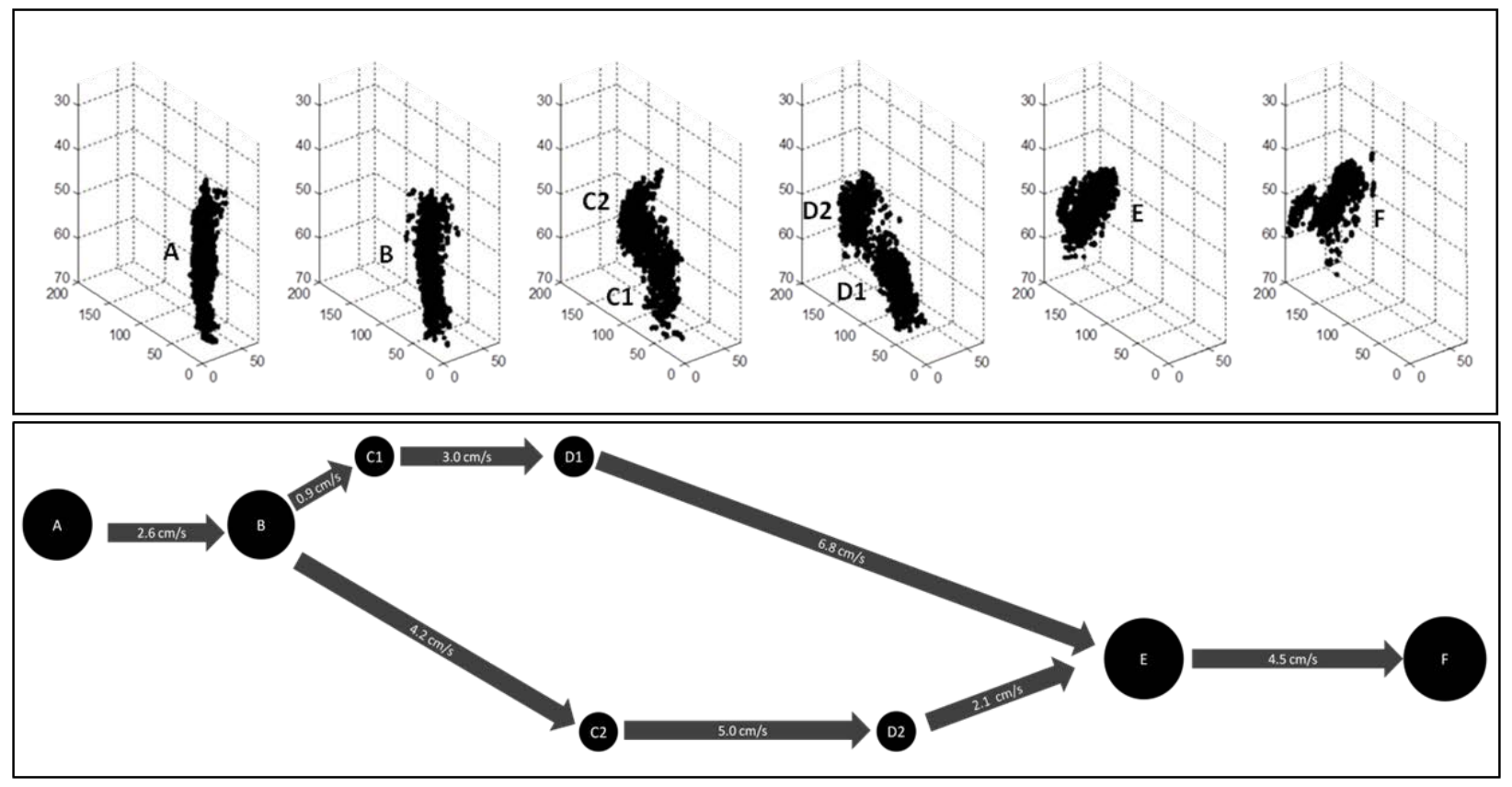

Figure 3. Anatomy of a fission (B to C1/C2) and fusion (D1/D2 to E) event. The upper panel shows raw detections extracted from six subsequent passes over an aggregation of fish with a multibeam echosounder data (5x vertical exaggeration). The lower panel describes the change in aggregation structure from pass to pass. In the lower panel, the size of the circles are proportional to the estimated number of fish, and the length of the arrows are proportional to the distance the school has moved. Data collected by the NOAA Alaska Fisheries Science Center acoustic/trawl walley pollock survey in the Gulf of Alaska, 2012. 